\title{
Aproximaciones teóricas sobre el rol de la publicidad electoral en contextos de malestar
}

\section{Theoretical approaches about the role of electoral advertising in a context of political malaise}

\author{
Pedro Fierro Zamora y Ana Azurmendi ${ }^{1}$ \\ Recibido: 20-03-2017 - Aceptado: 08-06-2017 \\ DOI: https://doi.org/10.26441/RC16.2-2017-A4
}

\begin{abstract}
RESUMEN: Desarrollaremos un marco teórico que nos permita comprender el rol que tendría la publicidad electoral en un contexto de malestar. Considerando la multidimensionalidad del descontento, entenderemos a la desafección como el componente que más se relaciona con aquello que Putnam y Goss denominan "las condiciones previas fundamentales para una democracia efectiva". En virtud de la evidencia sobre los efectos democráticos de las campañas, sugeriremos que la publicidad electoral nos podría ayudar a enfrentar el declive del sentido cívico de nuestros individuos desde su dimensión actitudinal, lo que tendría implicancias concretas en la calidad de nuestro sistema democrático.
\end{abstract}

Palabras clave: campañas electorales; desafección; actitudes políticas; democracia; sentido cívico.

\begin{abstract}
We are going to develop a theoretical frame to understand the role that campaigns would have in a context of political malaise. Considering the multidimensionality of the discontent, we are going to conceive the disaffection like the component more related with what Putnam \& Goss called 'the previous conditions for an effective democracy'. Taking into account the results of other studies surrounding the democratic effects of the campaigns, we will suggest that the electoral advertising could help us to improve the civic sense of the citizens from an attitudinal dimension, strengthening our democracies.
\end{abstract}

Keywords: political campaign; disaffection; political attitude; democracy; civic sense.

1 Pedro Fierro Zamora es Máster en Comunicación Política y Corporativa por la Universidad de Navarra. Imparte asignatura Opinión pública y liderazgo estratégico en la Universidad Adolfo Ibáñez y Escuela de Negocios en la ciudad Viña del Mar, Chile. pedro.fierro@uai.cl, http://orcid.org/0000-0002-8910-9461

Ana Azurmendi es Doctora por la Universidad de Navarra y Profesora Titular de Derecho de la Comunicación en la Facultad de Comunicación en la misma universidad. Imparte la asignatura Derecho de la Comunicación en los grados de Periodismo y de Comunicación Audiovisual. aazur@unav.es, http://orcid.org/0000-0001-66798826 


\section{Introducción}

Distintos estudios demoscópicos nos demuestran que la desconfianza y el descrédito de nuestras autoridades e instituciones siguen representando problemáticas comunes en las democracias contemporáneas (RodriguezVirgili, 2014). En esta línea, el presente trabajo tiene como objetivo principal desarrollar un marco teórico que nos permita comprender de mejor forma el rol de la publicidad electoral en el enfrentamiento de estos fenómenos asociados genéricamente al descontento ciudadano.

Para cumplir con el propósito declarado, realizaremos en primer lugar una revisión bibliográfica enfocada en la evolución histórica del malestar en las democracias consolidadas y emergentes. En esta línea, entenderemos que estos fenómenos implicarían un menoscabo del sentido cívico de nuestros ciudadanos, lo que se traduciría consecuentemente en un deterioro de la calidad del sistema democrático. Destacaremos, así, la necesidad de enfrentar el malestar en virtud de fortalecer lo que algunos autores denominan "el grado de democraticidad" de los países (O’Donnell, 2004).

De esta forma, y considerando la multidimensionalidad inherente al malestar, distinguiremos distintos elementos de la problemática, entendiendo finalmente que la desafección como dimensión específica del fenómeno - representa un aspecto subyacente a la buena calidad de la democracia. Sugeriremos que para comprender el verdadero valor democrático de la publicidad electoral se hace necesario estudiarla en virtud de sus efectos concretos en aquellas variables actitudinales usualmente asociadas a la desafección. Pese a que rescataremos importantes estudios que nos acercan a la comprensión de las consecuencias democráticas de los actos de campaña -aunque usualmente centrados en los aspectos conductuales y no actitudinales del involucramiento-, concluiremos que el tratamiento clásico que se le da a la problemática presenta algunas deficiencias conceptuales que impiden un desarrollo profundo al respecto. Estas limitaciones se verían profundizadas cuando analizamos la relación entre publicidad e involucramiento desde una perspectiva actitudinal.

\section{Fenómeno de malestar en democra- cias contemporáneas y el declive del sentido cívico: del asociacionismo toc- quevilliano al descontento político}

\subsection{Antecedentes históricos y concep- tuales del malestar: Estados Unidos como ejemplo de un fenómeno global}

Desde mediados del siglo $\mathrm{XX}$ ha existido un particular interés por el estudio de fenómenos relacionados con las conductas políticas de los individuos. En este sentido, parte de la literatura en ciencias políticas, sociología y comunicación pública se volcó al entendimiento de los procesos de descontento, generalizados muchas veces como malestar político (Offe, 2006; O'Keefe, 1980; Robinson, 1976; Torcal \& Montero, 2006). Más allá de 
los importantes avances en cuanto a la conceptualización y entendimiento de estos fenómenos, el malestar - como dimensión genérica - sigue siendo un objeto de estudio esencial a la hora de entender y analizar la participación ciudadana, esencialmente por las dificultades metodológicas y conceptuales que representa la problemática (Offe, 2006).

En términos históricos, el estudio del malestar comienza a tomar fuerza en la segunda parte del siglo XX (P. Abramson, 1983; Banducci \& Karp, 2003; Nye, Zelikow, \& King, 1997; Torcal \& Montero, 2006). Algunos autores entienden que en aquella época se produce una sobrecarga de los gobiernos, con el consecuente término de los periodos de riqueza y bienestar (Torcal \& Montero, 2006). Los ciudadanos esperaban de los gobiernos de turno resultados concretos que se adecuaran a los diversos intereses materiales e ideales de cada uno y, en la medida en que eso no sucedía, la consecuencia no era otra cosa que la frustración e insatisfacción por parte de la ciudadanía (Easton, 1976; Offe, 2006). Si bien la economía pareció estabilizarse con el correr de los años en los países industrializados, la desafección persistió. Así, en pleno triunfo del liberalismo, los países occidentales presentaban un alto grado de insatisfacción respecto al funcionamiento de la democracia representativa (Putnam \& Goss, 2003). Precisamente este descontento se ha llegado a considerar como uno de los principales desafíos de las sociedades contemporáneas (Schmitter, 1995).
De esta forma, los fenómenos de $\mathrm{ma}$ lestar no se presentaban como procesos abstractos, sino que más bien vinculados concretamente a un cambio en el comportamiento y sensaciones de los ciudadanos. Por este motivo, no se pueden entender estos procesos sin referirnos a la evolución del espíritu cívico en las democracias occidentales, considerando tanto su dimensión conductual como actitudinal.

Quien comprende de forma más lúcida esta temática es Alexis de Tocqueville (Offe, 2006; Pharr \& Putnam, 2000; Putnam, 1993; Skocpol, 1997). Si nos retrotraemos a la descripción que el francés hacía de Norteamérica en los años 1820', encontraremos una sociedad de un espíritu cívico envidiable y de gran interés por los asuntos públicos, lo que tenía distintas implicancias en términos conductuales (De Tocqueville, 2006 [1835]). En esta línea, el espíritu cívico descrito no se expresaba sólo en un fortalecido tejido social, sino que también en algunas conductas políticas convencionales. Quizás por eso la participación electoral de la mayor parte del siglo XIX alcanzaba el $75 \%$ al $90 \%$ en los Estados Unidos (Skocpol, 2003, p. 494).

Sin querer profundizar mayormente en la caracterización otorgada por el francés, lo relevante para nuestros efectos se relaciona con la permanencia de estas características durante gran parte del siglo XIX y del siglo XX. Al respecto, autores como Gabriel Almond $y$ Sidney Verba nos recuerdan que, al menos hasta los años 1960', el pueblo estadounidense seguía fuertemente 
comprometido e interesado en los asuntos públicos, lo que explicaba los altos índices de participación en organizaciones de voluntariado (Almond \& Verba, 1989, p. 313). Sin embargo, en la segunda mitad del siglo XX esta situación comienza a cambiar (P. Abramson, 1983; Banducci \& Karp, 2003; Craig, 1993). En cuanto a las conductas políticas convencionales, la participación electoral decrece (P. R. Abramson \& Aldrich, 1982; Shaffer, 1981), lo que se expande a la participación informal, donde - pese a la existencia de un gran número de organizaciones - la afiliación de los individuos disminuye considerablemente (Pharr \& Putnam, 2000; Wuthnow, 2003). De esta misma forma, los sindicatos también comienzan a verse menoscabados (Skocpol, 2003, p. 529). En definitiva, de la sociedad de fuerte espíritu cívico retratada por Tocqueville pasamos a una con redes más bien mermadas, con una afiliación envejecida y con una confianza en las instituciones que disminuía en el largo plazo (Wuthnow, 2003, p. 563).

Este declive de la cultura cívica estadounidense provocó importantes investigaciones centradas en el estudio del capital social (Coleman, 1990; Putnam, 1993, 1995), pero también tuvo implicancias importantes en el entendimiento de las actitudes hacia el sistema democrático y sobre las conductas políticas de los individuos en contextos de malestar. Sin embargo, nuestra intención en este capítulo no es centrarnos específicamente en el declive del capital social norteamericano, sino simplemente tomarlo como ejemplo de un fenómeno que se venía presentando a nivel global (Banducci \& Karp, 2003; Norris, 1999; Nye et al., 1997) y que afectaba directamente las percepciones ciudadanas respecto a las autoridades, instituciones $y$ sistemas democráticos.

\subsection{Del declive del espíritu cívico al descontento político}

En este sentido, Joseph Nye (1997) se atreve a concluir que en las últimas tres décadas del siglo XX la confianza en los gobiernos había decaído a nivel global (Nye et al., 1997). La lectura de Nye concuerda con la de algunos otros investigadores. Precisamente a partir de los setenta se comienza a entender que la sensación ciudadana de "impotencia, apatía e indiferencia hacia los políticos" empezaba a representar un fenómeno común en las democracias industrializadas (Easton, 1975, p. 456).

Con el objetivo de entender las percepciones de los individuos en contextos de malestar, podemos encontrar relevantes estudios centrados en el declive de la participación política - tanto formal como informal -, en la insatisfacción ciudadana, en la desconfianza respecto de las instituciones y en la falta de representatividad de los sistemas democráticos (Easton, 1975; Farah, Barnes, \& Heunks, 1979; Marsh \& Kaase, 1979; Miller, 1980; O’Keefe, 1980), pero siempre conscientes de que uno de los principales desafíos para los investigadores se relacionaba con tratar de dilucidar los elementos, las dimensiones, las causas y las consecuencias de estos procesos de descontento (Easton, 1976). Esta lectura resulta determinante para nuestros 
propósitos, pues si bien las conductas políticas representaban un foco de atención importante para la academia, se plantea a su vez la necesidad de estudiar sensaciones subyacentes, superiores y anteriores a las actuaciones individuales. De esta forma se entendían las preocupaciones de Nye (1997) y de Easton (1975), cuyas lecturas no apuntaban al declive de la participación propiamente tal, sino más bien a ciertas actitudes como la confianza - que podían ser variables explicativas de estos comportamientos.

Así, se comenzaba a estudiar la participación política desde una mirada genuinamente multidimensional, que entendía la existencia de distintas vías y opciones en virtud de formar parte activa del proceso democrático (Torcal \& Lago, 2006, p. 309). Era precisamente esto lo que implicaba reconocer que para cada conducta podían existir motivaciones y explicaciones diferentes, donde las sensaciones subyacentes tomarían un rol esencial (Marsh \& Kaase, 1979). Con todo esto, resultan algo más entendibles los planteamientos de Putnam y Goss (2003), quienes - a raíz del estudio del capital social estadounidense - nos hablaban de esas "condiciones previas fundamentales [...] para la existencia de una democracia efectiva" (Putnam \& Goss, 2003, p. 9).

Considerando la multidimensionalidad del malestar, el desafío será entonces determinar qué dimensión del fenómeno nos acercaría más a esas condiciones previas fundamentales, aunque antes de eso debemos referirnos a ciertos aspectos de aquella democracia efectiva.

\section{Calidad de nuestros ciudadanos y "democraticidad"}

\subsection{Aspectos generales sobre el buen ciudadano}

Como hemos señalado, los fenómenos de descontento - todavía mirados en términos genéricos - se relacionarían precisamente con ciertas actitudes y conductas que alejarían a los individuos de la cosa pública y que menoscabarían su sentido cívico. En otras palabras, con procesos que los harían “peores ciudadanos". Pero, ¿podemos hablar de buenos o malos ciudadanos? Si es así, ¿cómo definiríamos la calidad de nuestros ciudadanos?

Para Judith Shklar (1991) el buen ciudadano en democracia es alguien que participa de la vida política más allá del día de las elecciones, aquel informado y que hace valer su opinión para apoyar medidas justas y rechazar las injustas. Además, tiende a ser tolerante y con gran participación en la vida social comunitaria (Shklar, 1991, p. 5). Como podemos ver, la mirada de la autora tiene cierta similitud con la descripción tocquevilliana ya retratada en párrafos anteriores. En este orden, Offe (2006) nos ofrece una lectura de Shklar bastante interesante. En términos generales, señala que esta visión multidimensional del ciudadano ideal se podría reducir en dos grandes grupos de características. En primer lugar, uno más bien ligado a los motivos y causas que se pretenden promover en la vía pública por parte del individuo, los que se enmarcarían en una visión desarrollada del bien común. En segundo lugar, uno referido 
a la confianza del individuo en los mecanismos que ofrece el sistema político para que esas causas, motivaciones e intereses sean escuchados y procesados por el sistema de forma efectiva. Si seguimos esta línea, el intento de los ciudadanos por promover sus causas implicaría participación en los asuntos públicos, tanto a nivel político como social. Por otro lado, la percepción de que el sistema responde ante los intereses ciudadanos - y en particular los individuales - se relacionaría íntimamente con los indicadores de eficacia política externa, lo que algunos autores asocian al system responsiveness (P. R. Abramson \& Aldrich, 1982; Finkel, 1985), es decir, la sensación de que el proceso democrático es permeable y de que las acciones individuales pueden producir algún cambio relevante en el sistema (Campbell, Gurin, \& Miller, 1954).

De esta forma, el buen ciudadano estaría conformado por conductas - participación - y actitudes individuales hacia los asuntos públicos - interés, confianza o incluso eficacia política (Craig, Niemi, \& Silver, 1990)-, precisamente aquellos indicadores que han comenzado a decrecer con la aparición de los fenómenos de malestar desde hace ya más de tres décadas.

Con todo lo anterior, sería sensato sostener que estos procesos de descontento tienen incidencia directa en la calidad de nuestros ciudadanos - en cuanto afectan sus conductas y actitudes -, pero, ¿̇uáles serían las implicancias de esto?

En virtud de avanzar, lo que sigue en las próximas líneas responde a tres ideas concretas. En primer lugar, sostendremos que la calidad del ciudadano sería un elemento esencial de la calidad de la democracia. Luego, entenderemos que, pese a que existen elementos culturales que determinan la calidad de nuestros ciudadanos, también podemos encontrar dimensiones racionales - dependientes de la gestión y de la dirección propuesta por los incumbentes - que incidirían en el declive o fortalecimiento del espíritu cívico. De esta forma, consideraremos que, de estar genuinamente preocupados por mejorar la calidad de nuestra democracia, será esencial promover acciones destinadas a mejorar también la calidad de nuestros ciudadanos o, al menos, a elevar los indicadores relacionados con ello. Acá es donde la publicidad electoral tendría un rol especial.

\subsection{Calidad de nuestros ciudadanos como elemento esencial de los niveles de "democraticidad"}

Lo primero, entonces, será determinar por qué decimos que la calidad de nuestros ciudadanos representa un elemento esencial de la calidad de nuestra democracia.

Antes de entrar en el fondo de esta pregunta, no está de más reconocer que la cuestión de la calidad de la democracia representa una preocupación constante en la literatura. Sin embargo, independiente de la frecuencia de su uso, no siempre se determina su contenido. Por esta razón, preguntarnos por el estado de salud de los sistemas democráticos representa un esfuerzo interesante en un contexto de insatisfacción, cuando los focos de análisis se tienden a 
reducir casi por completo en el funcionamiento de la institucionalidad y en la promoción de la transparencia y probidad de nuestras autoridades.

Independiente de la metodología que sea utilizada para entender de mejor forma la calidad de nuestro sistema, existe cierto acuerdo respecto a que el régimen democrático no agota la democracia (Fernández-Llebrez, 2012; O'Donnell, 2003), es decir, que la extensión de esta última va mucho más allá del sistema político e institucional vigente. Esta visión no deja de ser sugerente para el contexto iberoamericano, pues, según el mismo Guillermo O'Donnell (2004), la pluralidad de factores que incidirían en el buen entendimiento del sistema se hacen aún más patente en las nuevas democracias. Probablemente una de las primeras ideas que haya que rescatar es que la democracia no representaría, entonces, un fenómeno binario, sino que más bien existirían distintos grados de "democraticidad" (O'Donnell, 2004). Así llegamos a un segundo punto, pues los elementos que determinarían el grado de "democraticidad" requerirían, entre otros factores, responder ante la promesa de que el poder soberano reside en los ciudadanos (Vargas Cullell, 2004). De esta forma, la civilidad de los individuos comienza a tomar una relevancia especial en el análisis.

Quizás es por esto que los elementos de la sociedad civil nunca han estado ausentes en los estudios sobre la calidad de nuestra democracia. Si consideramos por ejemplo el modelo inglés, el análisis contempla dimensiones ligadas a los derechos ciudadanos, a la representación y accountability de los gobiernos, a la dimensión internacional de la democracia y a la sociedad civil (Pineda, 2009). Si nos centráramos, por otro lado, en la metodología relacionada con los planteamientos de Guillermo O'Donnell - utilizada desde 1998 en contextos latinoamericanos -, la relevancia de la cultura cívica y la participación se exacerba aún más. De esta forma, no son pocos los autores que sostienen que la ciudadanía y la participación cívica debiesen ser estudiados como vectores de la calidad democrática (Fernández-Llebrez, 2012, p. 33). Es así precisamente como Jorge Vargas Cullel (2004), por ejemplo, declara que la sociedad civil requiere, entre otras cosas, apoyo en la creación de nuevas opciones para participar de los asuntos públicos, así como de mayores mecanismos para ejercer una labor de fiscalización frente a sus representantes (Vargas Cullell, 2004, p. 146).

En resumen, parece existir consenso respecto a que la democracia abarca distintas dimensiones y que la cultura cívica y la participación representan un elemento esencial al respecto.

Como hemos mencionado al analizar la propuesta de Shklar (1995) sobre el buen ciudadano, Offe (2006) entiende que las dos grandes variables que agrupan la multidemensionalidad propuesta se relacionan con el involucramiento político (political engagement) y con la eficacia política (sense of political efficacy), es decir, con conductas y actitudes. Respecto al involucramiento, lo planteado por Offe no simplifica del todo la 
problemática, pues la dimensión suele ser históricamente entendida de distintas formas por la literatura. Si bien hay algunos autores que lo relacionan con motivaciones más bien electorales (Conway, 2000) - como trabajar para partidos políticos o votar -, existe cierto acuerdo respecto a que las conductas políticas van algo más allá (P. Abramson, 1983; Almond \& Verba, 1989; Verba, Schlozman, \& Brady, 1995). Siguiendo esta línea, y sumando el hecho de que los planteamientos teóricos anteriormente formulado nos acercan a la lógica tocquevilliana de cultura cívica, entenderemos que las conductas a analizar debiesen contemplar la participación cívica y la política, ya sea esta última convencional (turnout) o no convencional. Por otro lado, la sensación de eficacia política se relaciona directamente con las actitudes individuales hacia el sistema político. Este indicador, junto al interés en política, la confianza en las instituciones, el negativismo y la apatía, representan variables populares en la literatura a la hora de medir los fenómenos de desafección (Austin \& Pinkleton, 1995; Marinus P. C. M. van Schendelen, 1981; Miller, 1980; O'Keefe, 1980; Pinkleton $\&$ Austin, 2002).

Con todo, ya parecen esclarecerse las razones por las cuales podríamos considerar la calidad de los ciudadanos considerando sus aspectos conductuales y actitudinales - como un elemento esencial de la calidad de los sistemas democráticos. Ahora, entonces, podríamos referirnos a los fundamentos culturales y contingentes que afectarían el sentido cívico de los individuos.

\subsection{Malestar como fenómeno cultural y racional}

Si bien hasta el minuto hemos hablado del malestar en términos genéricos, resulta a estas alturas evidente que estaría compuesto por dimensiones distintas, que responden a su vez de forma disímil ante diferentes variables (Torcal \& Lago, 2006). Esta primera idea resultará esencial a la hora de estudiar las eventuales causas y consecuencias de estos fenómenos.

Algunos investigadores han sostenido que cuando se habla de indicadores de malestar ligados a las condiciones previas fundamentales para el desarrollo de una democracia efectiva - en términos de Putnam y Gross - las causas estarían más bien ligadas a factores culturales de largo plazo, prescindiendo de elementos de la contingencia, que si bien pueden afectar la sensación de insatisfacción o el resultado de unas elecciones en particular, difícilmente afectarán las percepciones hacia el sistema, las instituciones y las autoridades en un sentido más profundo (Farah et al., 1979; Iyengar, 1980; Marsh \& Kaase, 1979).

Sin embargo, parte importante de la literatura disiente de esta postura (Banducci \& Karp, 2003; Hetherington \& Rudolph, 2008; Offe, 2006; Torcal \& Montero, 2006; Wuthnow, 2003). A propósito de las condiciones subyacentes a una buena democracia, Wuthnow (2003) sostiene, por ejemplo, que "la gestión de la nación puede crear (o erosionar) la confianza no sólo en el propio gobierno, sino también en las personas" (Wuthnow, 2003, p. 585). De esta misma forma, continúa sentenciando que "las consecuencias 
deberían estar claras para los líderes: si la población se halla menos dispuesta a confiar en la naturaleza humana no es porque dedique menos tiempo a participar en asociaciones voluntarias, sino porque los propios líderes no responden a sus expectativas" (Wuthnow, 2003, p. 585). Así, el autor entiende que las derivaciones del buen o del mal accionar de nuestras autoridades no se limitan a los grados de satisfacción que los individuos puedan tener con una determinada administración materializado en la evaluación de autoridades -, sino que también incidirían en otras variables más profundas de los ciudadanos, como la confianza. Con esta mirada, los escándalos políticos de distintos países iberoamericanos toman otro grado de relevancia. Si bien algunos análisis suelen centrarse en determinar cómo afectarían - o afectaron - la evaluación de autoridades o las próximas elecciones, lo que se nos plantea es que impactarían directamente en ciertas dimensiones subyacentes y esenciales a la hora de determinar la calidad de nuestros ciudadanos y de nuestra democracia.

Offe (2006), por su parte, también nos manifiesta que estos hitos pueden ser gatillantes de fenómenos que afectan tanto las conductas como las actitudes ciudadanas. En los términos que por el momento nos interesan, el autor plantea que los actos de corrupción terminan por desgastar la división entre el interés público y el privado - ya sea por comprar las decisiones públicas con fondos privados o por llenar con fondos públicos los bolsillos privados - incidiendo en el clima de desconfianza y apatía hacia la clase política (Offe, 2006, p. 36).
Todo esto es particularmente importante para nuestro análisis, pues tal como planteaba Wuthnow, así como la gestión de la nación puede erosionar la confianza, también puede crearla. Con esto se estaría sugiriendo que existen ciertas direcciones que nos permitirían superar los niveles actuales de apatía y cinismo, punto fundamental para entender posteriormente el rol que podría tener la publicidad electoral en un contexto de malestar.

Con todo lo anterior, lo que nos queda por concluir preliminarmente es que, cuando hablamos de las condiciones previas fundamentales para una democracia efectiva, podemos encontrar factores culturales -asociados al capital social- o racionales -vinculados a actos de corrupción o hitos de la contingencia-, los cuales no son excluyentes a la hora de explicar las causas de estos fenómenos de malestar (Torcal \& Montero, 2006).

Ahora, ya hemos mencionado que los fenómenos de malestar son multidimensionales y que contienen elementos diversos. Así también, declaramos que lo que nos interesa por el momento es aquella dimensión ligada a las condiciones subyacentes de una buena democracia. Pues bueno, ¿cómo identificar esas condiciones fundamentales entendiendo la complejidad del fenómeno?

\section{Malestar: la desafección como di- mensión específica subyacente}

\subsection{Insatisfacción, representatividad y desafección.}

Hasta el momento, hemos sostenido que desde la segunda mitad del siglo XX 
se han desarrollado fenómenos de malestar y descontento en gran parte de las democracias contemporáneas. Estos contextos se relacionarían con un declive del sentido cívico de los individuos, tanto desde la mirada de sus conductas - participación - como de las actitudes políticas hacia el sistema democrático. De esta misma forma, hemos entendido que este menguante espíritu cívico explicaría la baja calidad de nuestros ciudadanos, que a su vez podría ser entendida como dimensión esencial de la calidad de la democracia. Entonces, entendemos que los fenómenos de malestar - frutos tanto de la contingencia como de factores culturales - tienden a depreciar la calidad de nuestra democracia en virtud del deterioro de la calidad de nuestros ciudadanos. De esa forma, afirmábamos que de querer atacar los fenómenos de malestar - para mejorar la calidad de nuestra democracia deberíamos focalizarnos en desarrollar el espíritu cívico de nuestros ciudadanos, pero siempre desde una dimensión subyacente que nos acerque a esas condiciones previas fundamentales para una democracia efectiva de las que nos hablan Putnam y Goss.

Antes de profundizar en el rol que podría tener la publicidad electoral, el desafío será determinar qué dimensión del malestar nos acerca más a esos elementos subyacentes que buscamos. En este orden, distintos han sido los autores que han avanzado en el entendimiento de esta multidimensionalidad inherente al descontento ciudadano. Así, nos uniremos a aquella parte de la literatura que plantea una distinción clara entre lo que entendemos como insatisfacción, ilegitimidad y desafección (Offe, 2006; Torcal \& Montero, 2006), dimensiones que muchas veces suelen confundirse en la opinión pública.

En un primer término tenemos la insatisfacción, la que podría encontrar su fundamento en los planteamientos de Easton (1975) respecto al apoyo específico hacia las autoridades o instituciones. Lo que explica esta dimensión se relacionaría más bien con los intereses individuales de cada ciudadano y en el grado de protección que los incumbentes les otorgarían. De esta forma, el apoyo a un gobierno o las bajas evaluaciones de las autoridades se entenderían desde esta dimensión. Esto no deja de ser interesante, pues las direcciones implementadas en la gestión de los asuntos públicos, los escándalos o incluso los actos de corrupción podrían fácilmente relacionarse con altos índices de insatisfacción (Farah et al., 1979) - lo que se traduciría en bajos niveles de apoyo a las autoridades -, pero esto no necesariamente implicaría un declive del espíritu cívico de los ciudadanos en los términos que nos interesan.

Sin embargo, tal y como hemos sugerido, la insatisfacción no agotaría los fenómenos de malestar. El mismo Easton (1975) nos comentaba que algunas evaluaciones están íntimamente relacionadas con aquello que las autoridades políticas hacen y cómo las hacen, pero otras, en cambio, se encontrarían dirigidas hacia los "aspectos básicos del sistema" (Easton, 1975, p. 437). Con esto, distinguimos otra dimensión más bien relacionada con las percepciones ciudadanas 
respecto al régimen mismo: la sensación de ilegitimidad. Si la insatisfacción responde a los intereses de los individuos, la ilegitimidad responde a las creencias de los ciudadanos sobre el sistema democrático. Ya no hablamos de evaluación a autoridades, sino más bien del apoyo a la democracia en una dimensión más racional (Offe, 2006).

Sin embargo, la dimensión del malestar ligada a la sensación de ilegitimidad y al apoyo a la democracia como sistema de gobierno, tampoco nos acerca del todo a las condiciones subyacentes que buscamos. Tal como sucede con la insatisfacción, desconfiar de la democracia como régimen no implica necesariamente un desgaste del sentido cívico de los individuos.

Es acá cuando la desafección se muestra como una dimensión sobresaliente para nuestros intereses, sobre todo en virtud de que se trataría de un proceso que, si bien afecta las percepciones ciudadanas sobre autoridades e instituciones en términos difusos - sin importar quienes son los incumbentes -, no incidiría en el apoyo de los ciudadanos hacia el régimen democrático como sistema de gobierno (Nye et al., 1997; Rodriguez-Virgili, 2014).

\subsection{Desafección y las condiciones pre- vias fundamentales para una democra- cia efectiva}

Como ya hemos señalado, lo que nos interesa es determinar la dimensión del malestar más ligada a las condiciones previas fundamentales para una democracia efectiva. De esta forma, podremos identificar de mejor manera aque- llos indicadores subyacentes a la calidad de nuestros ciudadanos con el fin de buscar herramientas que nos permitan fortalecer la democracia.

Así, el marco conceptual que suele ser utilizado para trabajar la desafección se le atribuye a Di Palma (1970), entendiendo que se trataría del "sentimiento subjetivo de impotencia, cinismo y falta de confianza en el proceso político, los políticos y las instituciones democráticas que genera un distanciamiento y alienación respecto a éstos, y una falta de interés en la política y los asuntos públicos, pero sin cuestionar el régimen democrático" (citado en Torcal y Montero, 2006:6).

Como podemos ver, el entendimiento de la desafección se relacionaría en mayor medida con las actitudes ciudadanas hacia el sistema político, lo que se ve reflejado en la forma en que ha sido trabajada por distintos autores en términos empíricos. Así, y tal como hemos comentado, la eficacia política tanto interna como externa -, el interés en política, la confianza en las instituciones, el negativismo y la apatía, serían variables adecuadas a la hora de operacionalizar esta dimensión.

A diferencia de la insatisfacción o la ilegitimidad atribuida al sistema, la desafección como dimensión del malestar sí implicaría un menoscabo del sentido cívico de nuestros individuos, al afectar su dimensión actitudinal. De esta forma, las actitudes políticas se presentan como un elemento más profundo en la dinámica del malestar que la mala evaluación de nuestras autoridades o la baja participación de 
nuestros ciudadanos. Con todo lo anterior, sostenemos que sería la desafección aquella dimensión que más nos acercaría a las condiciones previas fundamentales de una buena democracia.

A raíz de los planteamientos de Thomas Petterson, Rodriguez-Virgili va en esta línea al sostener que la desafección "no sólo aumenta la abstención y disminuye la implicación ciudadana en las actividades de los partidos políticos, sino que los ciudadanos se muestran cada vez más distantes de los políticos y de la política" (Rodriguez-Virgili, 2014, p. 10). Esta postura es relevante en cuanto no se reduce a las actitudes, sino que también atribuye al fenómeno consecuencias negativas en términos de involucramiento. Si bien los efectos del malestar dependerán de muchos otros factores (Farah et al., 1979) - como los contextos y realidades de cada territorio -, se ha sostenido también que la desafección explicaría los bajos niveles de participación convencional y no convencional en las nuevas democracias, lo que profundizaría las brechas entre los ciudadanos y sus representantes (Torcal \& Lago, 2006). Precisamente esta fractura entre las estructuras de poder y los individuos explicaría algunos de los procesos que estamos viviendo en las democracias contemporáneas. $\mathrm{Al}$ respecto, uno de los principales patrones asociados a estos contextos se relaciona con el surgimiento de los políticos anti-políticos y con la explotación con fines estratégicos del miedo y la esperanza (Offe, 2006, p. 37).

Entonces, si la dimensión del malestar que pretendemos atacar - por ser la que más nos acerque a esas condiciones previas fundamentales para una democracia efectiva - es la desafección, y entendiendo que gran parte de la literatura la asocia a las actitudes de los ciudadanos hacia el sistema democrático, ¿qué herramientas disponemos institucionalmente para mejorar esas actitudes? ¿Podría ser la publicidad electoral un instrumento adecuado para combatir la desafección, mejorar así la calidad de nuestros ciudadanos y consecuentemente potenciar nuestra democracia?

\section{Campañas electorales en contextos de desafección}

Tal como hemos sugerido desde el inicio de este trabajo, consideramos que la publicidad electoral podría ser una herramienta interesante a la hora de combatir el actual clima de desafección por el que atraviesan distintas democracias contemporáneas. Sin embargo, lo que precisamente motiva este trabajo se relaciona con las distintas trabas que suelen existir a la hora de estudiar la problemática. Una de ellas sería precisamente la existencia de una conceptualización de las campañas históricamente deficiente por su visión restringida respecto a los efectos democráticos de estos actos.

En este sentido, Lippmann, por ejemplo, entendía las campañas como aquella actividad donde "se practica el arte de inducir a todo tipo de personas que piensan diferente a votar lo mismo" (Lippmann, 1922, p. 172). Por otra parte, Paul Lazarsfeld y su equipo las definiría como "el proceso de planificar y ejecutar actividades con la intención 
de ganar votos" (García \& D'Adamo, 2004, p. 7). Años más tarde, el profesor Christopher Arterton tampoco cambiaría el foco, al definirlas como aquellos "esfuerzos encaminados a dirigir mensajes persuasivos a un vasto electorado" (García \& D’Adamo, 2004, p. 7). Finalmente, y ya en tiempos contemporáneos, la profesora Pippa Norris las entendería como "esfuerzos organizados para informar, persuadir y movilizar" (Norris, 2002, p. 120).

Como podemos apreciar, desde la década del veinte el entendimiento de los actos de campaña suele compartir una estructura similar, en cuanto a que serían herramientas destinadas esencialmente a determinar la decisión y dirección del voto, tal como lo entenderían Campbell, Gurin y Miller (1952). Así, la conceptualización de Pippa Norris logra capturar gran parte de los avances en la materia, en cuanto a que los esfuerzos en el entendimiento de las campañas han estado, en su gran mayoría, centrados en determinar la forma en que persuaden, informan o comprometen al votante (Freedman, Franz, \& Goldstein, 2004; Goldstein \& Ridout, 2004; Huber \& Arceneaux, 2007; Lazarsfeld, Berelson, \& Gaudet, 1948), o lo que María José Canel entendería como lo cognitivo, afectivo y la acción (Canel, 1998).

Con todo, el hecho de que los actores que participan del proceso vean la problemática desde esta perspectiva de maximización de votos no es necesariamente el problema, sino más bien que el entendimiento académico y regulatorio se vea usualmente reducido a esta visión (López-Hermida Russo \& FierroZamora, 2016).

Desde un punto de vista democrático, ¿̇sólo sirven las campañas electorales para informar, persuadir y comprometer? Tomando en consideración las aproximaciones teóricas ya trabajadas, sugerimos que no, y para justificar esto podemos apoyarnos en distintos trabajos de investigación que tocan tangencialmente el tema.

La profesora Cristina Moreno, por ejemplo, entendía que la persuasión como elemento por excelencia buscado por sus promotores y patrocinadores es sólo una función de la publicidad electoral, existiendo también lo que ella denomina la legitimación y la selección de las elites políticas (Moreno, 2010). Basado en un estudio sobre las elecciones generales del 2008 en España, Moreno sugiere que el seguimiento de la información electoral - así como también las conversaciones sobre temas electorales en ese periodo - se relacionaba positivamente con los niveles de información política de los ciudadanos y con la probabilidad de votar (Moreno, 2010, p. 77). Si bien el foco no estaba puesto en los aspectos actitudinales del malestar, lo planteado por la investigadora tiene una gran importancia a la hora de estudiar los efectos democráticos de los actos electorales. Algo similar planteaba años antes la profesora Pippa Norris, quien aporta conclusiones esenciales a la hora de entender la relación de la publicidad electoral con el involucramiento cívico (Norris, 2001, 2002). Sin embargo, y tal como hemos comentado, tanto Norris como Moreno se enfocan 
en la información y la participación electoral como efectos de los actos de campaña, prescindiendo de otros elementos actitudinales subyacentes que a nosotros nos preocupan.

Con todo, y pese a que estos acercamientos son relevantes para nuestros propósitos, lo que nos interesa en definitiva es determinar el rol que tendrían las campañas y nuevas tecnologías en el fortalecimiento de aquellas condiciones previas fundamentales para una democracia efectiva, y que en los términos expuestos se acercarían a las actitudes políticas características a la hora de medir la desafección - en cuanto dimensión específica de los fenómenos de malestar -. Es acá donde investigaciones empíricas como la de Hansen y Tue Pedersen (2014) tienen un rol esencial. En un estudio reciente, los investigadores sugieren que las campañas no tan sólo incidirían en el aumento de los niveles de conocimiento político de nuestros ciudadanos - elemento ya tratado por Moreno y Norris -, sino que también en el mejoramiento de los índices de eficacia política, tanto interna como externa (Hansen \& Tue Pedersen, 2014).

En virtud de entender de mejor forma estos resultados, debemos recordar que eficacia política fue definida inicialmente como "la sensación de que la acción política individual puede, o no, impactar el proceso político" (Campbell et al., 1954, p. 187). Esta conceptualización, originalmente unidimensional, fue rápidamente entendida como un concepto con dos aspectos distintos. Sin pretender profundizar en el tema, la eficacia interna se relacionaría con la sensación de self-competence (Finkel, 1985) y la eficacia externa con lo que ya denominamos como system responsiveness (P. R. Abramson \& Aldrich, 1982). Considerando esto último, y tomando en consideración el enfoque de nuestro acercamiento, la eficacia política como objeto de estudio a la hora de medir los efectos democráticos de la publicidad electoral representa un tema particularmente interesante, en cuanto ya hemos sostenido que ha sido una de las actitudes políticas mayormente asociadas a la desafección (Marinus P. C. M. van Schendelen, 1981; Miller, 1980; O'Keefe, 1980; Pinkleton \& Austin, 2002; Torcal \& Montero, 2006). En este sentido, el valor de la investigación de Hansen y Tue Pedersen radica precisamente en que se aleja de los efectos en las urnas para comenzar a medir las implicancias de estas actividades de campaña en la conformación de una ciudadanía activa, más allá de la mera participación formal.

Lamentablemente, no encontramos una gran cantidad de estudios enfocados en los efectos democráticos de la publicidad electoral y, menos aún, centrados específicamente en estudiar los aspectos actitudinales del involucramiento político como variables dependientes. Esto no deja de ser curioso, pues cuando hablamos de la normativa sobre aspectos orgánicos de las votaciones sí que encontramos investigaciones que analizan sus efectos en los aspectos actitudinales del involucramiento (e.g. Banducci and Karp, 2003; Birch, 2010, 2008; Bowler and Donovan, 2002; Karp 
and Banducci, 2008; Norris, 2004), algo que también se replica cuando se analizan las consecuencias del proceso electoral como fenómeno político (Banducci \& Karp, 2003; Clarke \& Acock, 1989; Clarke \& Kornberg, 1992; Norris, 2002). Sin embargo, los elementos comunicacionales suelen ser analizados desde sus efectos en el resultado (Hansen \& Tue Pedersen, 2014, p. 303).

En resumen, y luego de esta revisión bibliográfica, entendemos que la conceptualización históricamente restringida de la publicidad electoral ha impedido avanzar en la materia. Estudios como el de Hansen y Tue Pedersen son aún incipientes y los impactos en el orden jurídico muchas veces inexistentes.

\section{Consideraciones finales}

Hemos sostenido que desde mediados del siglo XX se han comenzado a presentar fenómenos de malestar, los que se han expandido también a las nuevas democracias. Estos procesos incidirían, de acuerdo a la literatura, en el declive del espíritu cívico de nuestros ciudadanos, tanto en su dimensión actitudinal como conductual. De esta forma, consideramos también que estos fenómenos de malestar menoscababan el grado de "democraticidad" de los países, en cuanto afectaban uno de sus elementos esenciales: la calidad de sus ciudadanos. Con todo, resaltamos la necesidad de encontrar herramientas con las cuales pudiésemos enfrentar el malestar, pero asumiéndolo antes que todo como un fenómeno multidimensional. El desafío era determinar, en- tonces, aquella dimensión que más nos acercara a lo que Putnam y Goss denominan las condiciones previas fundamentales para una democracia efectiva. Acá es donde aparece la desafección como dimensión particular subyacente. Así, para entender el rol de la publicidad electoral en contextos de malestar, sería necesario centrarnos en los eventuales efectos que tendrían estos actos en los indicadores ligados a la desafección, usualmente cercanos a distintas actitudes políticas de los ciudadanos hacia el sistema democrático, como la confianza, el interés y la eficacia política.

En este contexto, consideramos que el restringido marco conceptual con que históricamente es estudiada la publicidad electoral ha incidido en los pocos avances en la materia, en cuanto a que la mayor parte de los esfuerzos sigue centrándose en los efectos que estos actos tendrían en la decisión y dirección del voto - o en lo que Hansen y Tue Pedersen denominan electoral outcome -, prescindiendo de sus implicancias en el proceso democrático desde un prisma más amplio.

De esta forma, el marco teórico trabajado sugiere la relevancia de ampliar la mirada respecto a la publicidad electoral en virtud de sus efectos generales en el sistema y en las actitudes subyacentes de nuestros ciudadanos, más allá de la mera participación electoral. Esto tendría una especial relevancia en contextos de malestar, presentes en gran parte de las democracias occidentales contemporáneas.

Consideramos que esta nueva conceptualización de la publicidad electoral 
tendría distintas implicancias en futuras investigaciones. Por ejemplo, nos obligaría a profundizar en la relación existente entre las distintas acciones electorales y el desarrollo de los aspectos actitudinales del involucramiento. De esta misma forma, un marco teórico enriquecido nos permitiría ampliar la literatura sobre el uso electoral de las nuevas tecnologías y la evolución del sentido cívico. Las implicancias de esta visión también se expandirían eventualmente a estudios jurídicos y normativos. Esto tiene un especial interés si consideramos que la propaganda electoral suele ser regulada más bien en función de la transparencia y equidad, perspectiva desde la que se tiende a la restricción más que a la promoción. Al considerar la afección como un eventual interés protegido por la legislación electoral, aspectos como la duración de las campañas, la difusión de nuevas tecnologías, la disposición de fondos públicos y el manejo de datos masivos debiesen ir tomando otro sentido y alcance para el legislador.

Con todo, las aristas académicas, jurídicas y políticas que se abren con un nuevo entendimiento de la publicidad electoral podrían ser tan variadas como interesantes, lo que justifica la necesidad de avanzar en la materia desde una dimensión empírica, teórica y normativa.

\section{Referencias Bibliográficas}

Abramson, P. (1983). Political Attitudes in America: Formation and Change. W.H.Freeman \& Co Ltd.

Abramson, P. R., \& Aldrich, J. H. (1982). The Decline of Electoral Participation in America. The American Political Science Review, 76(3), 502-521. https:/doi. org/10.2307/1963728

Almond, G. A., \& Verba, S. (1989). The Civic Culture. Political Attitudes and Democracy in Five Nations. Sage.

Austin, E. W. \& Pinkleton, B. E. (1995). Positive and negative effects of political disaffection on the less experienced voter. Journal of Broadcasting E Electronic Media, 39(2), 215-235. https://doi.org/10.1080/08838159509364300

Banducci, S. A. \& Karp, J. A. (2003). How Elections Change the Way Citizens View the Political System: Campaigns, Media Effects and Electoral Outcomes in Comparative Perspective. British Journal of Political Science, 33(3), 443-467.

Birch, S. (2008). Electoral institutions and popular confidence in electoral processes: A crossnational analysis. Electora Studies, 27, 305-320.

Birch, S. (2010). Perceptions of Electoral Fairness and Voter Turnout. Comparative Political Studies, 43(12), 1601-1622. https://doi.org/10.1177/0010414010374021

Bowler, S. \& Donovan, T. (2002). Democracy, Institutions and Attitudes about Citizen Influence on Government. British Journal of Political Science, 32(2), 371-390.

Campbell, A. Gurin, G., \& Miller, W. E. (1954). The voter decides. Row, Peterson \& Co.

Canel, M. J. (1998). Los efectos de las campañas electorales. Comunicación Y Sociedad, 11(1), $47-67$. 
Clarke, H. D. \& Acock, A. C. (1989). National Elections and Political Attitudes: The Case of Political Efficacy. British Journal of Political Science, 19(4), 551-562.

Clarke, H. D. \& Kornberg, A. (1992). Do National Elections Affect Perceptions of MP Responsiveness? A Note on the Canadian Case. Legislative Studies Quarterly, 17(2), 183204. https://doi.org/10.2307/440057

Coleman, J. S. (1990). Foundations of Social Theory. The Belknap Press of Harvard University Press.

Conway, M. M. (2000). Political Participation in the United States (3th ed.). Washington DC: Congressional Quarterly.

Craig, S. C. (1993). The Malevolent Leaders: Popular Discontent In America (Political Cultures). Westview Press.

Craig, S. C., Niemi, R. G., \& Silver, G. E. (1990). Political Efficacy and Trust: A Report on the NES Pilot Study Items. Political Behavior, 12(3), 289-314.

De Tocqueville, A. (2006). Democracia en America. Fondo de Cultura Económica.

Easton, D. (1975). A Re-Assessment of the Concept of Political Support. British Journal of Political Science, 5(4), 435-457.

Easton, D. (1976). Theoretical Approaches to Political Support. Canadian Journal of Political Science / Revue Canadienne de Science Politique, 9(3), 431-448.

Farah, B., Barnes, S. H., \& Heunks, F. (1979). Political Dissatisfaction. In S. H. Barnes \& M. Kaase (Eds.), Political Action. Mass Participation in Five Western Democracies (pp. 409-447). Beverly Hills, CA: Sage.

Fernández-Llebrez, F. (2012). Humanismo, participación y ciudadanía cívica. Elementos para una democracia de calidad. Revista Española de Ciencia Política, 30(Nov), 31-53.

Finkel, S. E. (1985). Reciprocal Effects of Participation and Political Efficacy: A Panel Analysis. American Journal of Political Science, 29(4), 891-913. https://doi.org/10.2307/2111186

Freedman, P., Franz, M., \& Goldstein, K. (2004). Campaign Advertising and Democratic Citizenship. American Journal of Political Science, 48(4), 723-741. https://doi. org/10.2307/1519930

García, V., \& D’Adamo, O. (2004). Campañas electorales y sus efectos sobre el voto: Análisis de la campaña electoral presidencial 2003 en Argentina. Psicología Política, 28, 7-25.

Goldstein, K., \& Ridout, T. N. (2004). Measuring the effects of televised political advertising in the United States. Annual Review of Political Science, 7(1), 205-226. https://doi. org/10.1146/annurev.polisci.7.012003.104820

Hansen, K. M., \& Tue Pedersen, R. (2014). Campaigns Matter: How Voters Became Knowledgeable and Efficacious During Election Campaigns. Political Communication, 31 , 303-324.

Hetherington, M. J., \& Rudolph, T. J. (2008). Priming, Performance, and the Dynamics of Political Trust. The Journal of Politics, 70(2), 498-512. https://doi.org/10.1017/ s0022381608080468

Huber, G. A., \& Arceneaux, K. (2007). Identifying the Persuasive Effects of Presidential Advertising. American Journal of Political Science, 51(4), 957-977. 
Iyengar, S. (1980). Subjective Political Efficacy as a Measure of Diffuse Support. Public Opinion Quarterly, 44(2), 249-256. https://doi.org/10.1086/268589

Karp, J. A., \& Banducci, S. A. (2008). Political Efficacy and Participation in Twenty-Seven Democracies: How Electoral Systems Shape Political Behaviour. British Journal of Political Science, 38(2), 311-334.

Lazarsfeld, P., Berelson, B., \& Gaudet, H. (1944). The people's choice: How the voter makes up his mind in a presidential campaign. Columbia University Press.

Lippmann, W. (1922). Opinión Pública. Editorial C. de Langre.

López-Hermida Russo, A. P., \& Fierro-Zamora, P. (2016). Campañas políticas y desafección ciudadana: aproximación desde Chile a los efectos de las actividades electorales en el proceso democrático. Palabra Clave, 19, 365-397.

Marinus P. C. M. van Schendelen. (1981). Disaffected Representation in the Netherlands: A Nonaffected Reappraisal. Political Behavior, 3(2), 137-162.

Marsh, A., \& Kaase, M. (1979). Measuring Political Action. In S. H. Barnes \& M. Kaase (Eds.), Political Action. Mass Participation in Five Western Democracies. Beverly Hills, CA: Sage (pp. 57-96)

Miller, W. E. (1980). Disinterest, Disaffection, and Participation in Presidential Politics. Political Behavior, 2(1), 7-32.

Moreno, C. (2010). El efecto de la campaña para las elecciones generales españolas de 2008 sobre la información política y la participación electoral de los votantes: ¿se puede hablar de una función de legitimación de las campañas electorales? Revista Española de Ciencia Política, 24, 53-81.

Norris, P. (1999). Critical Citizens: Global Support for Democratic Governance. Oxford University Press.

Norris, P. (2001). Un círculo virtuoso? El impacto de las comunicaciones políticas en las democracias post-industriales. Revista Española de Ciencia Política, 4(1), 7-33.

Norris, P. (2002). Do political campaigns matter? Campaign effects in elections and referendums. In D. M. Farrell \& R. Schmitt-Beck (Eds.), Do campaign communications matter for civic engagement? American elections from Eisenhower to George Bush. Routledge.

Norris, P. (2004). Electoral Engineering: Voting Rules and Political Behavior. Cambridge University Press.

Nye, J., Zelikow, P. D., \& King, D. C. (1997). Why People Don't Trust Government. Harvard University Press.

O’Donnell, G. (2003). Democracia, desarrollo humano y derechos humanos. In G. O'Donnell, J. Vargas Cullell, \& O. M. Iazzetta (Eds.), Democracia, desarrollo humano y ciudadanía: reflexiones sobre la calidad de la democracia en América Latina (pp. 25-148). Rosario, Argentina: Homo Sapiens.

O'Donnell, G. (2004). Human Development, Human Rights, and Democracy. In G. O'Donnell, J. Vargas Cullell, \& O. M. Iazzetta (Eds.), The quality of democracy: theory and applications (pp. 9-92). Indiana: University of Notre Dame Press. 
Offe, C. (2006). Political disaffection as an outcome of institutional practices? Some post-Tocquevillean speculations. In M. Torcal \& J. R. Montero (Eds.), Political Disaffection in Contemporary Democracies. Routledge.

O’Keefe, G. J. (1980). Political Malaise and Reliance on Media. Journalism \& Mass Communication Quarterly, 57(1), 122-128. https://doi.org/10.1177/107769908005700118

Pharr, S., \& Putnam, R. D. (2000). Disaffected Democracies: What's Troubling the Trilateral Countries? Princeton University Press.

Pineda, C. (2009). Recensiones. Revista de Estudios de La Administración Local Y Autonómica, $311,305-316$.

Pinkleton, B. E., \& Austin, E. W. (2002). Exploring Relationships Among Media Use Frequency, Perceived Media Importance, and Media Satisfaction in Political Disaffection and Efficacy. Mass Communication and Society, 5(2), 141-163. https://doi.org/10.1207/ S15327825MCS0502_3

Putnam, R. D. (1993). Making democracy work: Civic traditions in modern Italy. Princeton University Press.

Putnam, R. D. (1995). Tuning In, Tuning Out: The Strange Disappearance of Social Capital in America. PS: Political Science and Politics, 28(4), 664-683. https://doi. org/10.2307/420517

Putnam, R. D., \& Goss, K. A. (2003). Introducción. In R. D. Putnam (Ed.), El declive del capital social (pp. 9-33). Galaxia Gutenberg Círculo de Lectores.

Robinson, M. J. (1976). Public Affairs Television and the Growth of Political Malaise: The Case of "The Selling of the Pentagon." The American Political Science Review, 70(2), 409-432.

Rodriguez-Virgili, J. (2014). Comunicación para combatir la desafección política. In A. Motta (Ed.), Grandes espacios de la comunicación política. Construyendo nuevos ideales (Vol. 1). México: Universidad Panamericana.

Schmitter, P. C. (1995). More Liberal, Preliberal, or Postliberal? Journal of Democracy, 6(1), $15-22$.

Shaffer, S. D. (1981). A Multivariate Explanation of Decreasing Turnout in Presidential Elections, 1960-1976. American Journal of Political Science, 25(1), 68-95. https://doi. org/10.2307/2110913

Shklar, J. (1991). American Citizenship. The Quest for Inclusion. Cambridge: Harvard University Press.

Skocpol, T. (1997). The Tocqueville Problem: Civic Engagement in American Democracy. Social Science History, 21(4), 455-479. https://doi.org/10.2307/1171662

Skocpol, T. (2003). América cívica, pasado y presente. In R. D. Putnam (Ed.), El declive del capital social (pp. 491-543). Galaxia Guttenberg Círculo de Lectores.

Torcal, M., \& Lago, I. (2006). Political participation, information, and accountability: some consequences of political disaffection in new democracies. In M. Torcal \& J. R. Montero (Eds.), Political Disaffection in Contemporary Democracies. Social Capital, Institutions, and Politics (pp. 308-332). London: Rutledge. 
Torcal, M., \& Montero, J. R. (2006). Political Disaffection in Comparative Perspective. In M. Torcal \& J. R. Montero (Eds.), Political Disaffection in Contemporary Democracies. Social Capital, Institutions, and Politics (pp. 3-20). Rutledge.

Vargas Cullell, J. (2004). Democracy and the Quality of Democracy: empirical findings and methodological and theoretical issues drawn from the citizen audit of the quality of democracy in Costa Rica. In G. O’Donnell, J. Vargas Cullell, \& O. M. Iazzetta (Eds.), The Quality of Democracy (pp. 93-162). Indiana: University of Notre Dame Press.

Verba, S., Schlozman, K. L., \& Brady, H. E. (1995). Voice and Equality. Civic Voluntarism in American Politics. Cambridge: Harvard University Press.

Wuthnow, R. (2003). El carácter cambiante del capital social en Estados Unidos. In P. Robert (Ed.), El declive del capital social. Galaxia Guttenberg Círculo de Lectores. 